\title{
Measurement of Gas Mixture Using Field Asymmetric Ion Mobility Spectrometry
}

\author{
Yasufumi Yokoshiki ${ }^{1}$, Takamichi Nakamoto ${ }^{1}$ \\ 1 Tokyo Institute of Technology, Nakamoto Lab., R2-5, 4259 Nagatsuta-cho, Midori-ku, Yokohama, \\ Kanagawa 226-8503, Japan
}

\begin{abstract}
:
The performance of Field Asymmetric Ion Mobility Spectrometry (FAIMS) was investigated for the vapor mixtures. We measured the binary mixture composed of ethanol and acetone. The head space above the binary mixture liquid was introduced into FAIMS. Although the fingerprint originating from ethanol was not initially observed, it gradually appeared as time increased. It suggests that there is a possibility to obtain the feature of the mixture composition.
\end{abstract}

Key words: FAIMS, Odor, Mixture Compounds, Changing Concentrations, Separation Measurement

\section{Introduction}

Since odor reproduction has a variety of applications, we have so far studied it [1]. For this purpose, the fundamental and important technique is the mixture quantification. Although we previously studied QCM sensor array, the collinearity problem should be overcome when the number of ingredients increases. Although the mass spectrometry is appropriate for this purpose, a typical mass spectrometer does not work neither in the gas phase nor in the real time. Thus, we focused on Field Asymmetric Ion Mobility Spectrometry(FAIMS) [2]. In this report, we observed the response pattern of the mixture to check the possibility of mixture quantification.

\section{Experimental System}

Figure 1 shows block diagram of our experimental system. OLP-EK-023 (AtonARP,
JAPAN) was used as FAIMS device. Air was used as carrier gas supplied by the compressor (PC3-10HL, YAEZAKI KUATSU, JAPAN). A clean filter (CF3-02, IAC, JAPAN), an air dryer (AD3-02, IAC, JAPAN) and a carbon filter (3001-17201, GL science, JAPAN) were also used to remove the impurities in the air. Flow rate and Pressure were adjusted to 2 SLM, 1 bar respectively using regulator (RC200-PR20L4, CHELIC, Taiwan) and mass flow controller (MFC) (SEC-400MK3, HORIBA, JAPAN). The air was divided into two lines; one was connected to a bottle with odor from the headspace. The flow rate in the bottle was controlled by MFC and set to 50 SCCM. The two lines were mixed again and were supplied to FAIMS device through an inlet filter (T300A025A, Toyo Roshi Kaisha, JAPAN). FAIMS was controlled using the software on PC.

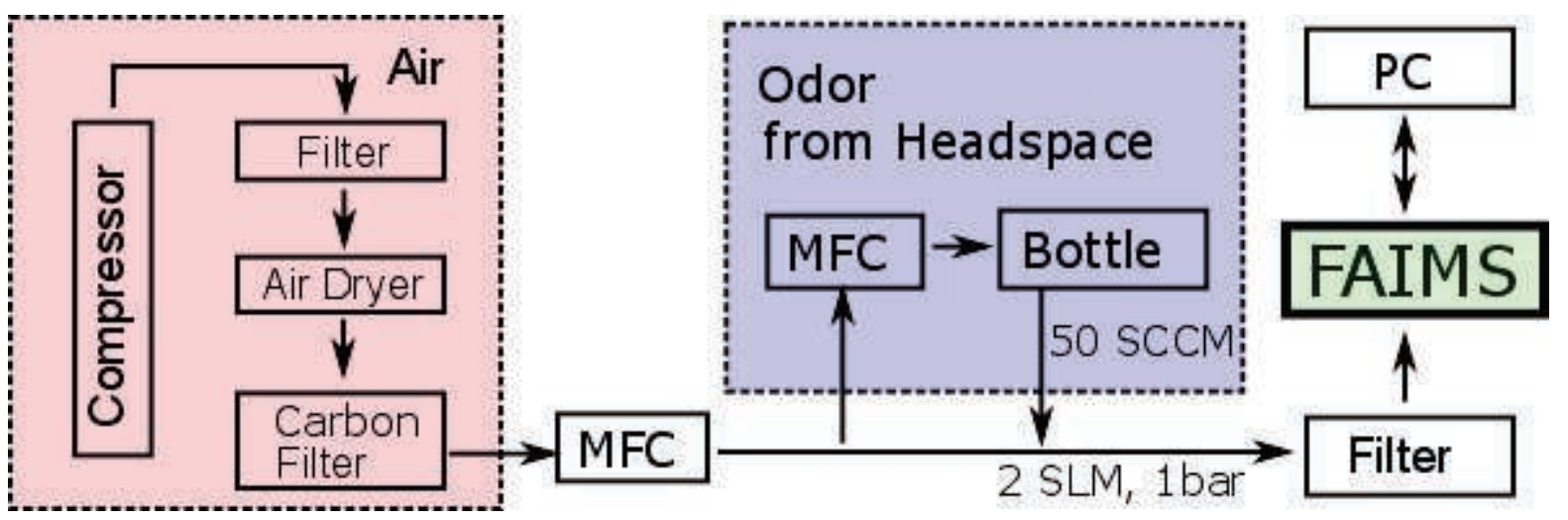

Fig. 1 Block Diagram of the Experimental System 


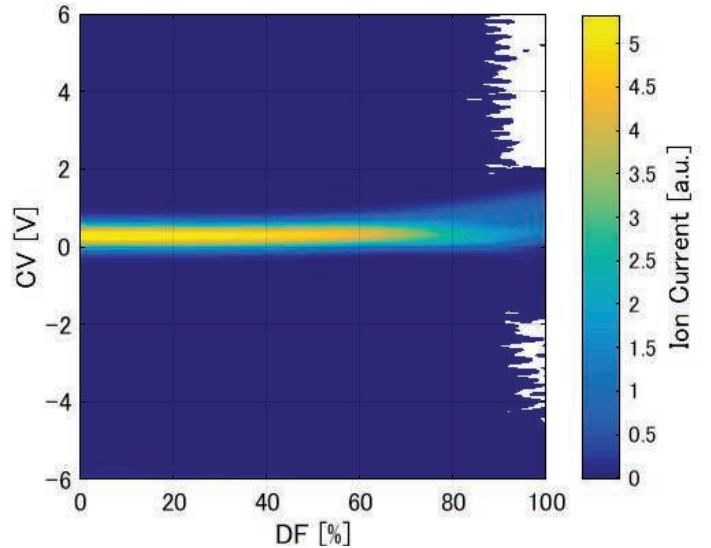

Fig. 2 Measurement Results of Ethanol(10 ul) and Acetone (5 ul) in Water $(1 \mathrm{ml})$.

\section{Experiment and Results}

First, we investigated the relationship between concentration of single substance and the output. Ethanol and acetone were selected as targets. It turned out that output did not change so much even though the concentration change was large. Next, we measured mixture of ethanol and acetone. Ethanol (10ul) and acetone (5ul) was also dissolved in $1 \mathrm{ml}$ of water (See Fig. 2). In FAIMS, Ion is separated by the electric field. Horizontal axis represents relative electric field strength (DF, dispersion field). Vertical axis means bias voltage between the electrodes ( $\mathrm{CV}$, compensation voltage). Although the fingerprint changed as compared with the single compound case, initially no peak specific to ethanol was found (See Fig. 3, portion A) and increasing peak was also found (See Fig. 4, portion B). Therefore, we observed only the peak ( $\mathrm{DV}=80 \%$ ), and how it changes with time (See Fig. 5). Figure 5 shows ethanol peak appeared after a certain time. It was found that the peak originating from ethanol did not appear initially, however the ethanol peak location gradually shifted and then peak appeared (See Fig. 5, portion C).

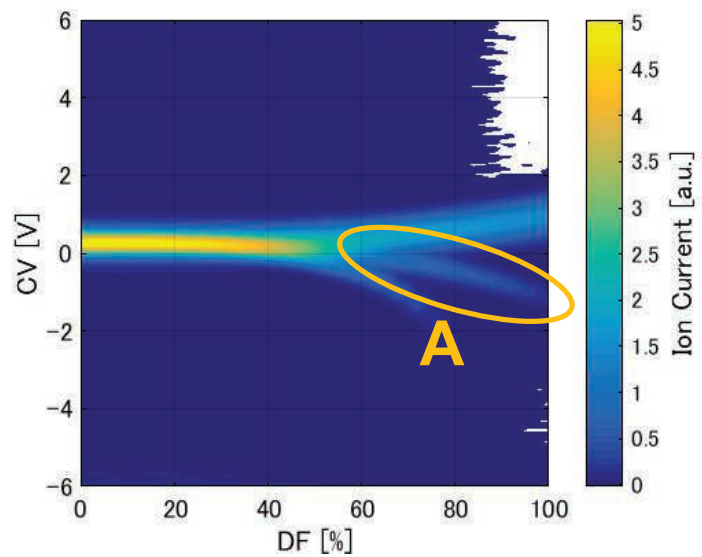

Fig. 3 Measurement Results of Ethanol(10 ul) in Water $(1 \mathrm{ml})$.

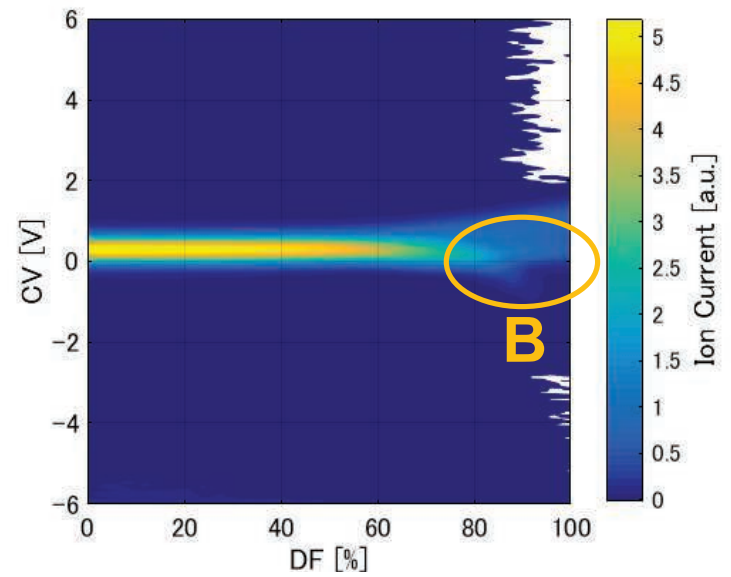

Fig. 4 Measurement Results of Acetone(5 ul) in Water $(1 \mathrm{ml})$.

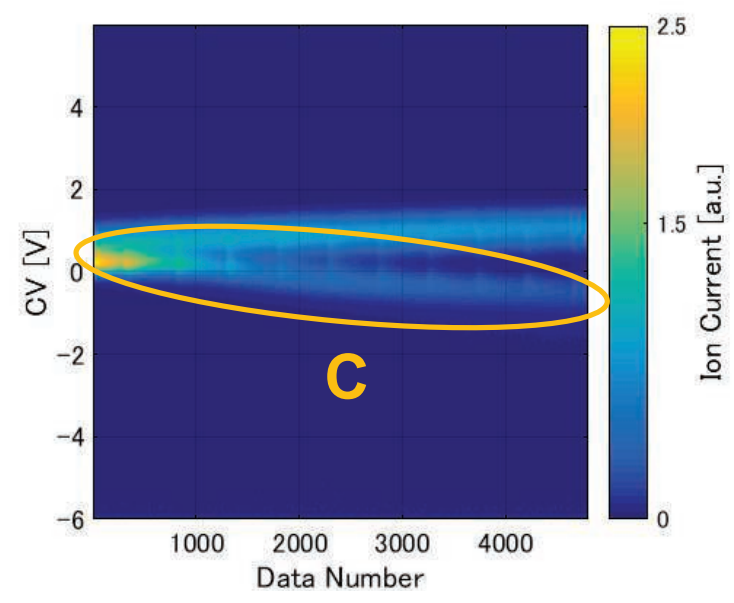

Fig. 5 Measurement Results of Ethanol(10 ul) and Acetone $(5 \mathrm{ul})$ in Water $(1 \mathrm{ml})$. DV $=80 \%$

It indicates that the mixture composition in the liquid phase gradually changes since ethanol evaporation speed is different from acetone's one. Moreover, acetone was more easily ionized than ethanol. That is why only acetone peek was observed at first and then ethanol peak appeared later.

\section{Conclusion}

In this study, it was found that it was useful for analyzing gas mixture compounds using FAIMS. It was also useful to consider the movement of the peak location. This results suggest that there is a possibility for the mixture analysis.

\section{References}

[1] T. Nakamoto, Introduction to Essentials of Machine Olfaction and Tastes, Wiley, 247314(2016); doi: 10.1002/9781118768495.ch1

[2] J. Li et al., Using Field Asymmetric Ion Mobility Spectrometry for Odor Assessment of Automobile Interior Components, IEEE Sensors Journal, vol. 16, no. 14, 5747- 5756(2016); doi: 10.1109/JSEN.2016.2568209 